Transport

Volume 171 Issue TR1

Pedestrian roundabout improvement strategy in subway stations

Sun, Hao, Gong, Qiu and Chen

ice | proceedings
Proceedings of the Institution of Civil Engineers Transport 171 February 2018 Issue TR1 Pages 20-29 https://doi.org/10.1680/jtran. 16.00073 Paper 1600073 Received 08/04/2016 Published online 23/03/2017

Keywords: rail \& bus stations/traffic engineering/ transport management ICE Publishing: All rights reserved
Accepted 08/02/2017

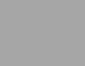

ice

$\overline{\text { Institution of Civil Engineers }}$

\title{
Pedestrian roundabout improvement strategy in subway stations
}

Lishan Sun PhD

Associate Professor, Beijing Key Laboratory of Traffic Engineering, Beijing University of Technology, Beijing, P. R. China

(corresponding author: Issun@bjut.edu.cn)

Siyuan Hao MSc

Graduate Assistant, Beijing Key Laboratory of Traffic Engineering, Beijing University of Technology, Beijing, P. R. China

\section{Qingsheng Gong MSc}

Graduate Assistant, Beijing Key Laboratory of Traffic Engineering,

Beijing University of Technology, Beijing, P. R. China

\section{Shi Qiu PhD}

Assistant Professor, Beijing Key Laboratory of Traffic Engineering, Beijing University of Technology, Beijing, P. R. China

\section{Yanyan Chen PhD}

Professor, Beijing Key Laboratory of Traffic Engineering, Beijing University of Technology, Beijing, P. R. China

Intersecting streams of pedestrians are frequently seen in subway stations. When two or more such streams encounter each other in the normal way, there is interference of progress and a reduction in speed. This research explores the impact of roundabout design on intersecting pedestrian streams by conducting a series of experiments on pedestrians in the field. First, the impact of roundabout size on different volumes of pedestrian traffic is examined; second, the effect of adding guiding signs is investigated; and third, a case where a greater number of streams intersect is studied. Results indicate that, although placing a roundabout at pedestrian intersections reduces the total space available and lengthens the average walking distance, the speed of pedestrian streams increases. The experiment reveals that the larger the radius of the roundabout, the more significant is the increase in speed and the smaller is the dispersion of acceleration. In addition, the role of the roundabout becomes more important as pedestrian traffic volume increases. Furthermore, the addition of guiding signs before the roundabout entrance is helpful in terms of regulating the direction of traffic and reducing possible conflict. Finally, the experiments reveal that inserting a roundabout also provides benefits in the case of three intersecting pedestrian streams.

\section{Introduction}

Because of the aggregation effect at transfer stations in subways, intersection of multiple pedestrian streams is commonly seen. These intersecting streams apparently cause pedestrian traffic conflicts and reduce travel speed, which is clearly undesirable when organising the transit of passengers through subways. However, they are practically unavoidable, even when the planning and design of pedestrian schemes has been reasonably well thought out, as it is unrealistic to expect to be able to use bridges or traffic lights for the purpose of separating these streams, unlike the case for intersecting vehicular traffic flow. In a recent survey carried out by the present research team, it was found that 43 out of the 46 stations investigated had two-stream pedestrian intersections. Also, of the 46 stations, 12 had threestream intersections and five stations had intersections that involved more than three streams of pedestrians.

Ando et al. (1988) were forerunners in terms of investigating two crossing pedestrian streams in a subway station. They observed the macroscopic behaviour of pedestrians intersecting at an angle. However, the characteristics of intersecting streams have mostly been based on qualitative observations or simulation studies in the past (Helbing, 1996, 1998; Helbing and Molnar, 1998). A few researchers have pointed out that unstable roundabout traffic can be formed when streams bound in different directions intersect, which appears like a dummy roundabout set at the position of the pedestrian intersection (Duparcmeur et al., 1995; Helbing, 1997; Molnar, 1996). The difference when compared with actual roundabouts is that the direction of rotation of the circular pedestrian streams was alternating (Helbing et al., 2001). Although traffic around roundabouts has to make a small detour, the frequency of necessary deceleration and stopping is found to decrease, thereby considerably reducing the potential for conflicts (Ando et al., 1988; Helbing and Molnar, 1998). In addition, the style of the roundabout - for example, turbo roundabouts, flower roundabouts or target roundabouts - has been studied (Mauro, 2010; Tollazzi et al., 2015a, 2015b). In order to stabilise roundabout traffic, Helbing et al. (2001) proposed that planting a column or a tree in the middle of a crossing could suppress the phases of 'vertical' or 'horizontal' motion in the area of the intersection. By conducting computerised simulations, the efficiency of transit speed of the pedestrian stream was demonstrated to be increased by up to $13 \%$.

Self-organisation provides another focus for the study of pedestrians. The self-organisation phenomenon of a stripe formation in pedestrian intersecting streams has been extensively observed (Ando et al., 1988; Dzubiella and Löwen, 2002; Hoogendoorn and Daamen, 2005; Hoogendoorn and Piet, 2003; Usher and Strawderman, 2008). Pedestrian stripes, like lanes on the highway, are a phenomenon of segregation. When pedestrian streams coming from two different directions intersect, pedestrians moving in the same direction become distributed in stripes (Dzubiella and Löwen, 2002). Within the same stripe, the pedestrians' walking speed and travel direction are usually identical (Hoogendoorn and Piet, 2003). Indeed, the 
Pedestrian roundabout improvement

strategy in subway stations

Sun, Hao, Gong, Qiu and Chen stripe formation phenomenon is even more clearly observed when the intersecting angle is $90^{\circ}$ (Hoogendoorn and Daamen, 2005). Based on the stripe formation phenomenon, providing a circular barrier at an intersection was assumed to form more marked and coherent stripes, and the circular barrier was found to perform better than a square barrier and no barrier in terms improvement of the efficiency of motion (Helbing et al., 2005).

A review of the literature suggests that the insertion of roundabouts would benefit traffic at intersections and should therefore be encouraged. However, the existing studies on pedestrian roundabouts have mostly been based on theoretical research or computerised simulations, which can neither be validated nor reflect the characteristics of pedestrian behaviour in the real world. To obtain more quantitative characteristics of intersecting streams, experiments on pedestrians are an extensively used method (Guo et al., 2010; Helbing et al., 2005; Wong et al., 2010; Xie et al., 2013). In a study by Guo et al. (2010), speed and density were analysed for two intersecting streams. The passing time of two intersecting streams has been used to analyse the self-organisation effect (Helbing et al., 2005).
In controlled experiments involving two pedestrian streams, macroscopic parameters such as speed, density and flow were extracted to calibrate the model of bidirectional pedestrian streams (Wong et al., 2010; Xie et al., 2013).

In the present research, the feasibility and effects of adding a roundabout for intersecting streams are explored using a series of experiments on pedestrians in the field. The methodology is given in the following section, and then the rest of the paper is organised into four sections. Section 3 investigates the impact of roundabout settings on the pedestrian stream. Section 4 evaluates the function of traffic guiding signs at roundabouts. The previous studies are then expanded to look at a threestream intersection in Section 5. Finally, some conclusions and recommendations are provided.

\section{Methodology}

A series of pedestrian experiments was conducted in a courtyard of Beijing University of Technology (BJUT) on the afternoon of 17 April 2015. The experimental scenarios were established according to the scheme shown in Figure 1. To mimic the two intersecting streams, two entrances and exits

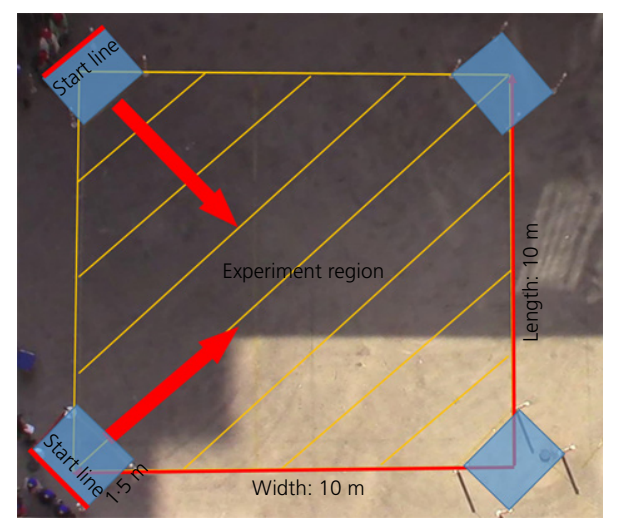

(a)

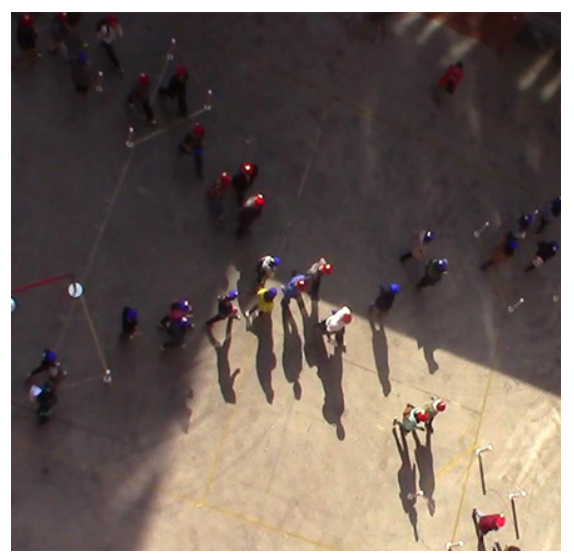

(b)

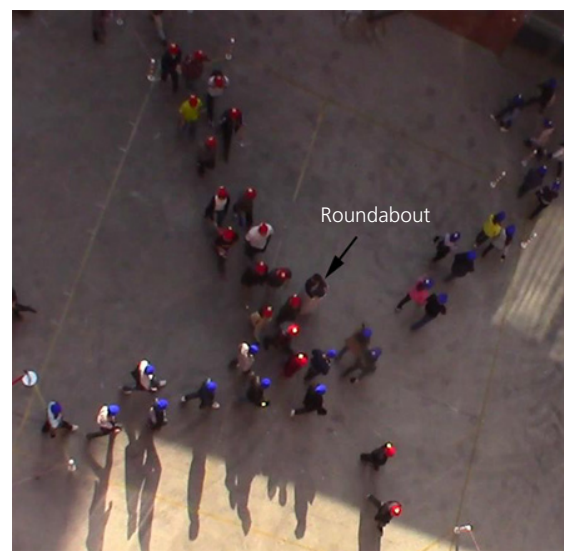

(c)

Figure 1. (a) Division into regions of the experimental scenario. (b) No-roundabout experimental scenario. (c) Experimental scenario with roundabout inserted 
Table 1. Comparison of experiment and real environment

\begin{tabular}{|c|c|c|c|c|c|}
\hline \multirow[b]{2}{*}{ Comparison of scenario } & \multicolumn{2}{|c|}{ Scenario setting } & \multicolumn{3}{|c|}{ Significance of pedestrian characteristic factors } \\
\hline & Volume & Number of pedestrians & Transit time & Speed & Acceleration \\
\hline Scenario 1 & 1350 & 25 & $0 \cdot 22$ & 0.13 & $0 \cdot 31$ \\
\hline Scenario 2 & 2050 & 25 & $0 \cdot 10$ & $0 \cdot 11$ & $0 \cdot 17$ \\
\hline Scenario 3 & 4800 & 50 & 0.12 & 0.08 & $0 \cdot 12$ \\
\hline
\end{tabular}

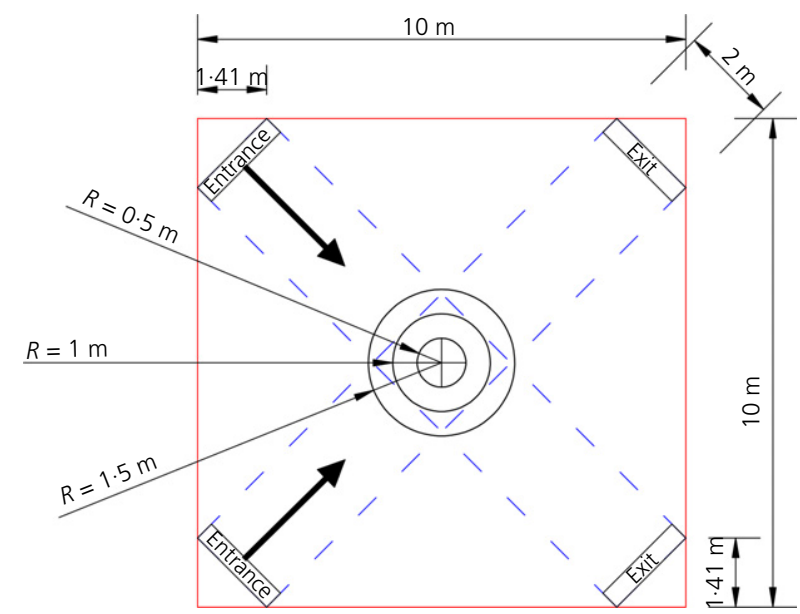

Figure 2. Experimental design of roundabout at two intersecting pedestrian streams

were designed. Considering the layout of the subway, 50 healthy undergraduate students from BJUT were recruited for the experiment. The number of participants was enough to develop several different experiments. Bearing in mind that any acquaintance among the students could lead to the production of biased experimental results, the participants were selected from different classes and departments. The ages of the participants ranged from 18 to 25 years old. Caps of different colours were used to distinguish the different streams. As can be seen in Figure 1(a), to obtain a normal flow without being affected by any starting delay, start lines positioned $1.5 \mathrm{~m}$ away from each entrance were drawn. To analyse the pedestrian behaviour in an automated way, a digital camera was placed at a height of $20 \mathrm{~m}$ above the experimental area. The digital camera had a resolution of $1920 \times 1080$ pixels and the capture rate was 30 frames/s, which is adequate to record pedestrian behaviour (Sun et al., 2014). The field experiment scenes (screenshots from the experimental video) are shown in Figures 1(b) and 1(c).

As a data-processing tool, SIMI Motion (a software application that uses novel algorithms to process video footage) was used for motion capture in this study (Sun et al., 2014). Pedestrians were labelled by using the SIMI Motion software. The position $(x, y)$ of each pedestrian was then captured automatically by SIMI Motion every $0.2 \mathrm{~s}$; the output also included speed, time, acceleration and so on.
Table 2. Experimental scenario settings of different roundabout designs

\begin{tabular}{|lcc|} 
Scenario & $\begin{array}{c}\text { Radius of } \\
\text { roundabout: } \mathbf{m}\end{array}$ & $\begin{array}{c}\text { Volume of pedestrian } \\
\text { traffic: }(\mathbf{p} / \mathbf{h}) / \mathbf{m}\end{array}$ \\
\hline Scenario 1a & - & 4000 \\
Scenario 1b & - & 5000 \\
Scenario 1c & - & 6000 \\
Scenario 2a & 0.5 & 4000 \\
Scenario 2b & 0.5 & 5000 \\
Scenario 2c & 0.5 & 6000 \\
Scenario 3a & 1.0 & 4000 \\
Scenario 3b & 1.0 & 5000 \\
Scenario 3c & 1.0 & 6000 \\
Scenario 4a & 1.5 & 4000 \\
Scenario 4b & 1.5 & 5000 \\
\hline
\end{tabular}

Table 3. Walking distance and transit times for the different experiments conducted

\begin{tabular}{|c|c|c|c|c|}
\hline $\begin{array}{l}\text { Radius of } \\
\text { roundabout: } m\end{array}$ & None & 0.5 & 1.0 & $1 \cdot 5$ \\
\hline Volume: $(p / h) / m$ & \multicolumn{4}{|c|}{ Walking distance: $\mathrm{m}$} \\
\hline 4000 & 11.95 & $12 \cdot 82$ & 13.07 & $14 \cdot 34$ \\
\hline 5000 & $12 \cdot 16$ & $12 \cdot 49$ & $12 \cdot 98$ & 14.83 \\
\hline 6000 & $12 \cdot 03$ & $12 \cdot 32$ & $13 \cdot 00$ & $15 \cdot 07$ \\
\hline & \multicolumn{4}{|c|}{ Passing time: $\mathrm{s}$} \\
\hline 4000 & 28 & 27 & 24 & 27 \\
\hline 5000 & 24 & 22 & 21 & 23 \\
\hline 6000 & 20 & 21 & 17 & 20 \\
\hline
\end{tabular}

Before the formal experiments were carried out, three trial experiments were conducted to compare the coherence of pedestrian characteristic factors between the pedestrian flow in the experiment and actual pedestrian flow in a subway. Video footage from a real subway hallway with intersecting pedestrian streams was analysed using SIMI Motion. Trial experiments were conducted according to the traffic volume/number of pedestrians obtained from these subway videos. One-way analysis of variance (Anova, where $p<0.05$ represents a significant difference and $p>0.05$ represents no significant difference) was conducted for the three factors of passing time, speed and acceleration and the significance is shown in Table 1. In this study, the passing time was taken as the total time from the first person entering the experiment area until the last person walked out of the experimental area. Results indicate that there was no 
Table 4. Descriptive statistics of speed under different traffic volumes (STD, standard deviation)

\begin{tabular}{|c|c|c|c|c|c|c|}
\hline Volume of pedestrian traffic: $(p / h) / m$ & Radius of roundabout: $m$ & Mean: $\mathrm{m} / \mathrm{s}$ & Medium: $\mathrm{m} / \mathrm{s}$ & Max.: $\mathrm{m} / \mathrm{s}$ & Min.: $\mathrm{m} / \mathrm{s}$ & STD: $\mathrm{m} / \mathrm{s}$ \\
\hline \multirow[t]{4}{*}{4000} & None & $1 \cdot 18$ & $1 \cdot 16$ & $1 \cdot 72$ & 0.57 & 0.40 \\
\hline & 0.5 & $1 \cdot 26$ & $1 \cdot 21$ & $1 \cdot 70$ & 0.63 & 0.38 \\
\hline & 1.0 & 1.29 & $1 \cdot 26$ & 1.74 & 0.60 & 0.32 \\
\hline & 1.5 & $1 \cdot 30$ & $1 \cdot 27$ & 1.85 & 0.66 & 0.24 \\
\hline \multirow[t]{4}{*}{5000} & None & 1.09 & 1.08 & 1.70 & 0.42 & 0.47 \\
\hline & 0.5 & $1 \cdot 18$ & $1 \cdot 10$ & 1.74 & 0.64 & 0.41 \\
\hline & 1.0 & 1.23 & $1 \cdot 16$ & 1.82 & 0.62 & $0 \cdot 30$ \\
\hline & 1.5 & 1.25 & $1 \cdot 16$ & 1.73 & 0.65 & $0 \cdot 16$ \\
\hline \multirow[t]{4}{*}{6000} & None & 0.89 & 0.85 & 1.72 & 0.40 & 0.49 \\
\hline & 0.5 & 0.99 & 0.85 & 1.69 & 0.42 & 0.33 \\
\hline & 1.0 & 1.08 & 1.03 & 1.86 & 0.66 & 0.29 \\
\hline & 1.5 & $1 \cdot 13$ & 1.07 & 1.65 & 0.61 & 0.23 \\
\hline
\end{tabular}

significant difference between the trial experiments and the realworld subway hallway. This finding demonstrates that the experiment could mimic a real-world scenario.

\section{Investigating impact of roundabout settings on pedestrian streams}

\subsection{Design of pedestrian experiment}

The aim in this section is to investigate the impact of different roundabout designs on intersecting pedestrian streams. Three aspects are explored: $(a)$ whether the existence of the roundabout has an impact on a weaving stream and, if so, what this impact is; $(b)$ the influence of the size of the roundabout on intersecting pedestrian streams; and $(c)$ the performance of the roundabout under different volumes of pedestrian traffic. Hence, experiments consisting of different combinations of pedestrian traffic volumes and roundabout sizes are developed.

According to the Transportation Research Board's (TRB's) Transit Cooperative Research Program (TCRP) Report 100, the Transit Capacity and Quality of Service Manual, the standard one-way passageway capacity is 5000 (persons $/ \mathrm{h}$ )/m (unit $(\mathrm{p} / \mathrm{h}) / \mathrm{m}$ is used in the rest of this paper) (Kittelson \& Associates et al., 2003). In this study, as any potential conflict may vary according to different traffic volumes, three levels of pedestrian volumes were investigated: $4000(\mathrm{p} / \mathrm{h}) / \mathrm{m}, 5000(\mathrm{p} / \mathrm{h}) / \mathrm{m}$ and $6000(\mathrm{p} / \mathrm{h}) / \mathrm{m}$, indicating that there were six, seven and eight experimental participants randomly assigned to a row, respectively. The experimental stream ratio is $1: 1$ in this study. Based on this assumption, three sizes of roundabout were designed, of radius $0.5 \mathrm{~m}, 1.0 \mathrm{~m}$ and $1.5 \mathrm{~m}$, as seen in Figure 2. Thus, in total, 12 scenarios were designed and the experiments were conducted for analysis, as listed in Table 2.

\subsection{Data analysis}

\subsubsection{Walking distance and transit time}

Three pedestrian experiments were conducted for each scenario and the average measurement was taken from these. Table 3 shows the average walking distance and transit time under

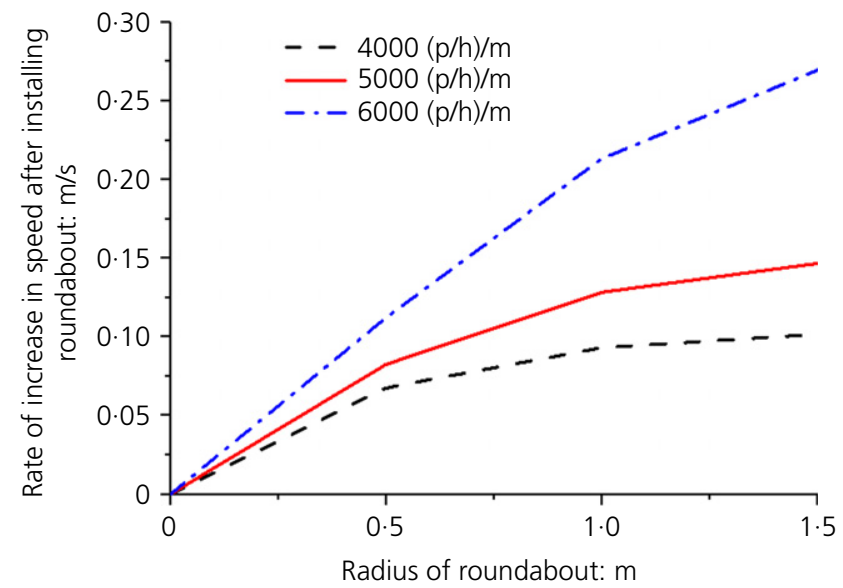

Figure 3. Rate of increase in mean speed after installing roundabout

different combinations of traffic volume and roundabout settings. It was found that for the same size roundabout the average walking distances for different traffic volumes vary little, which demonstrates that the pedestrian volume has barely affected the route selection. However, with the increase of roundabout size, the average walking distance increases. This is evident because the pedestrians have to walk around the roundabout to reach their destinations. The larger the roundabout is, the larger is the detour distance and hence the total walking distance.

As regards transit time, the comparison between a 'no-roundabout' scenario and scenarios with different sizes of roundabout is of interest. When the roundabout's radius is $0.5 \mathrm{~m}$, the change in path of movement has little effect on travel distance and, therefore, there is only a small change in transit time. A maximum of $2 \mathrm{~s}$ change is observed for the three different volumes. When the roundabout's radius is increased to $1.0 \mathrm{~m}$, an average of about $3-4 \mathrm{~s}$ drop in transit time is seen, which is about $15 \%$ reduced when compared with the no-roundabout scenario. However, when the roundabout's radius is increased to $1.5 \mathrm{~m}$, where the walking distance is the largest, the transit time is less than or the 


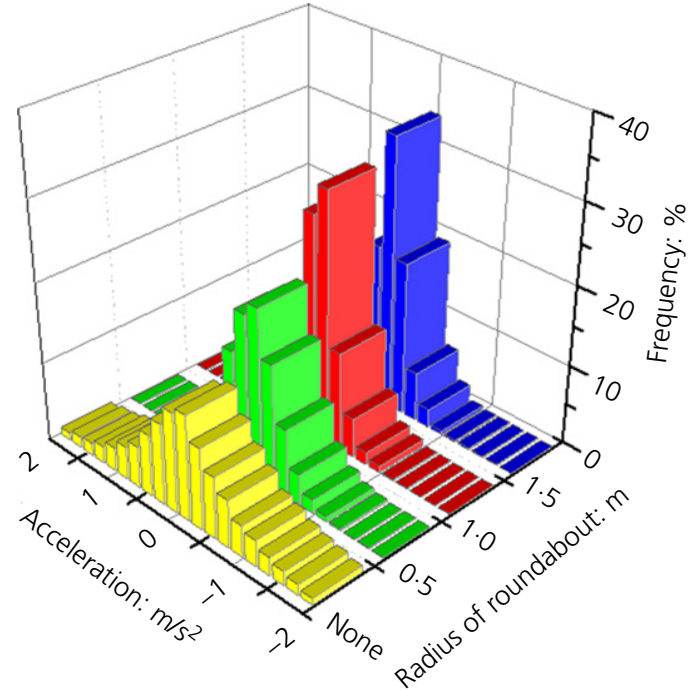

(a)

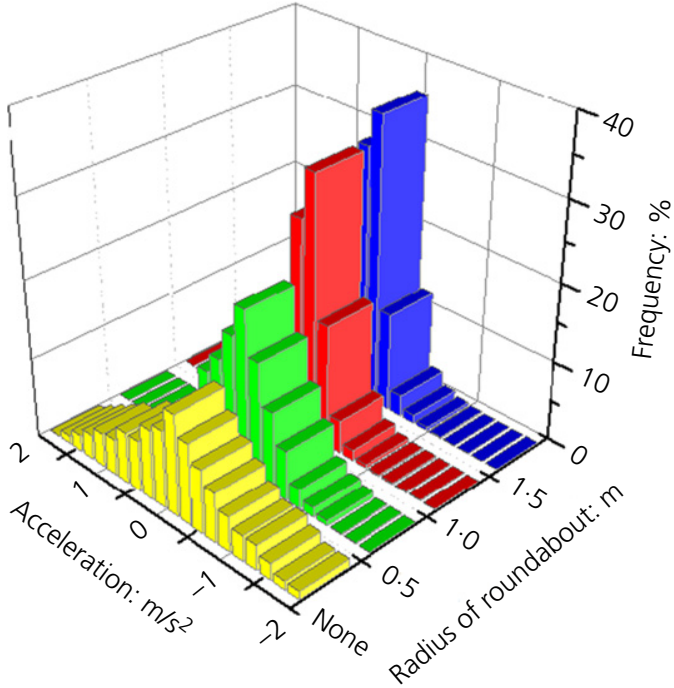

(b)

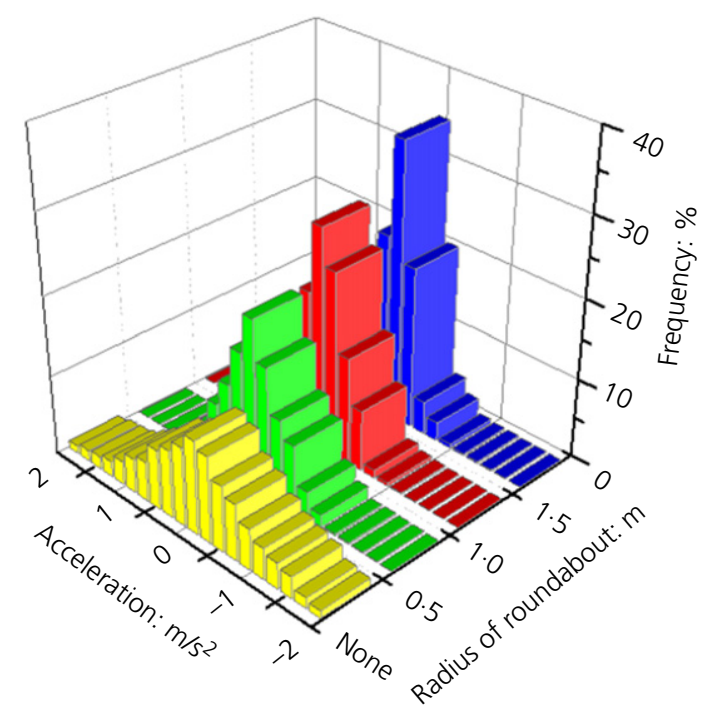

(c)

Figure 4. Frequency of acceleration in pedestrian traffic volume of: (a) 4000 (p/h)/m; (b) 5000 (p/h)/m; (c) 4000 (p/h)/m

same as the situation where no roundabout has been added. This observation suggests that a large roundabout has some positive effect on walking for intersecting streams. Overall, although walking distance increases when a roundabout is inserted, the transit time decreases. Thus, the transit efficiency of whole pedestrian streams increases. It should also be noted that, because the number of pedestrians in each experiment is the same, the transit time decreases with increase of pedestrian traffic volume.

\subsubsection{Speed}

The descriptive statistics regarding speed under different volumes of pedestrian traffic are listed in Table 4. Compared with the scenarios without a roundabout, the installation of a roundabout leads to an increase in pedestrian speed and a decrease in the standard deviation of speed for all traffic volumes. This phenomenon indicates that the installation of a roundabout could improve the traffic condition in general. For an identical traffic volume, the mean and median speeds increase and the standard deviation decreases with the expansion of the roundabout radius. However, this observation does not support a conclusion that the larger roundabout is, the better the pedestrian walking condition is. The size of roundabout could not be infinitely large, and should depend on the size of the area of intersection.

To investigate the influence of roundabout size on pedestrian speed under different traffic volumes, the rate of increase of 
mean speed is analysed in Figure 3. It can be seen that the greater the experimental traffic volume is, the greater is the rate of increase in speed. It is clear that the rate of increase in pedestrian mean speed at $6000(\mathrm{p} / \mathrm{h}) / \mathrm{m}$ is significantly higher than that for the other pedestrian traffic volumes.

\subsubsection{Acceleration}

Frequent acceleration and deceleration is one of the hazard factors in vehicular traffic; it is also undesirable in pedestrian traffic (Hediyeh et al., 2012). The acceleration data were extracted to investigate the influence of roundabout installation on change in speed. Figure 4 shows the distribution of pedestrian acceleration for different traffic volumes and roundabout installations. It is apparent that, after installing the roundabout, a more centred distribution of acceleration is found for all traffic volumes. This demonstrates that installing a roundabout could effectively reduce the occurrence of changes in acceleration.

In summary, the installation of a roundabout is beneficial to intersecting traffic. However, the improvement depends on the size of the roundabout. In the three sizes of roundabouts used in the current study, the largest one at $1.5 \mathrm{~m}$ radius shows the best overall traffic condition (no significant increase in transit time, but higher speed and less acceleration). In addition, the improvement in speed is the most significant at the largest experimental pedestrian traffic volume $(6000(\mathrm{p} / \mathrm{h}) / \mathrm{m})$.

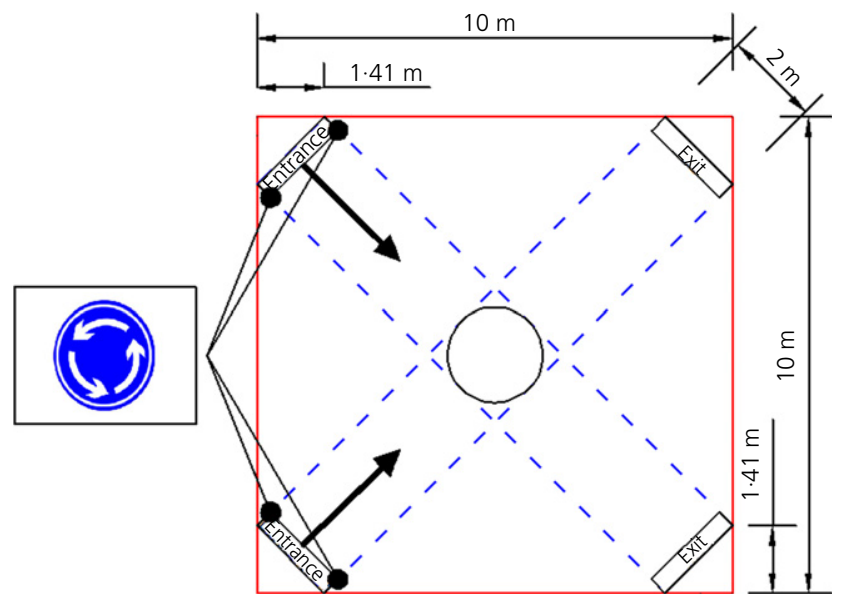

Figure 5. Experimental scenarios with traffic-guiding signage added

\section{Evaluating the effect of traffic guiding signage}

\subsection{Design of pedestrian experiment}

After a roundabout has been installed, pedestrians can walk around it in either a clockwise or counterclockwise direction. In highway regulation, only one-way traffic is allowed at roundabouts. Guiding signs are installed in front of roundabouts to direct drivers. This study also introduced guiding signage for pedestrian management at roundabouts. According to the law and custom in China, cars and pedestrians should travel on the right-hand side of thoroughfares. Therefore, signage to direct users in a counterclockwise direction was installed in front of the roundabout (Figure 5). A separate experiment was conducted to observe the effect of the traffic sign. In this experiment, a pedestrian volume of $5000(\mathrm{p} / \mathrm{h}) / \mathrm{m}$ and roundabout radius of $1.0 \mathrm{~m}$ were employed.

\subsection{Data analysis}

Speed, acceleration, walking distance and transit time as derived from the new experiments (shown in Table 5) are compared with those derived from scenario $3 \mathrm{~b}$. Figure 6 shows the pedestrian walking tracks for both experiments. Each line represents a pedestrian's walking track. Gradients are given according to speed and represented by symbols, where solid circles indicate the lowest speed and open squares the highest (see key). The speed can represent the degree of conflict between pedestrian traffic flows. The lower the speed is, the more severe is the conflict. Overall, the positions of lower speed are mostly distributed in and before intersecting areas, especially those areas where there are the most serious conflicts of pedestrian traffic.

It is evident that the traffic guiding signs elicited a walking distance that was increased by $5 \cdot 70 \%$. From Figure 6 it can be seen that almost all of the pedestrians walk in a counterclockwise direction, which leads to detours for some pedestrians. However, the transit time is decreased by $14 \cdot 28 \%$ compared with the scenario without guiding signs. In addition, the average speed is increased and the standard deviation is decreased by $3 \cdot 25 \%$ and $26 \cdot 70 \%$, respectively. With respect to acceleration, a $12 \cdot 20 \%$ reduction of standard deviation can be observed for the scenario with signs. All the parameters point to the finding that guiding signage plays a positive role in improving traffic efficiency at roundabouts.

In particular, the conflict points, as represented on Figure 6, are worth noticing. Without the guidance of the traffic signs, pedestrians would choose either to turn left or right at the

Table 5. Parameters analysis under different traffic guiding signage

\begin{tabular}{|lccccc|}
\hline Traffic guiding sign & Transit time: $\mathbf{s}$ & Walking distance: $\mathbf{m}$ & Mean of speed: $\mathbf{m} / \mathbf{s}$ & STD of $\mathbf{s p e e d : ~} \mathbf{m} / \mathbf{s}$ & STD of acceleration: $\mathbf{m} / \mathbf{s}^{\mathbf{2}}$ \\
\hline No signs (new experiment) & 21 & 12.98 & 1.23 & 0.30 & 0.41 \\
Signs included (scenario 3b) & 18 & 13.72 & 1.27 & 0.22 & 0.36
\end{tabular}




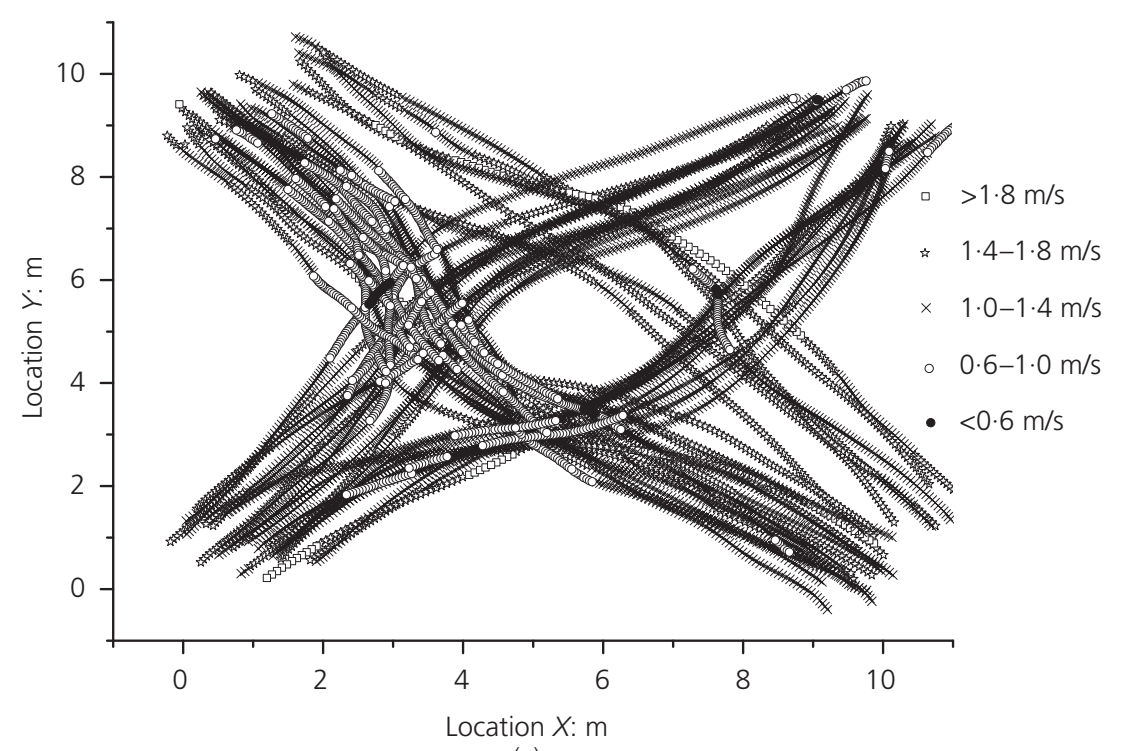

(a)

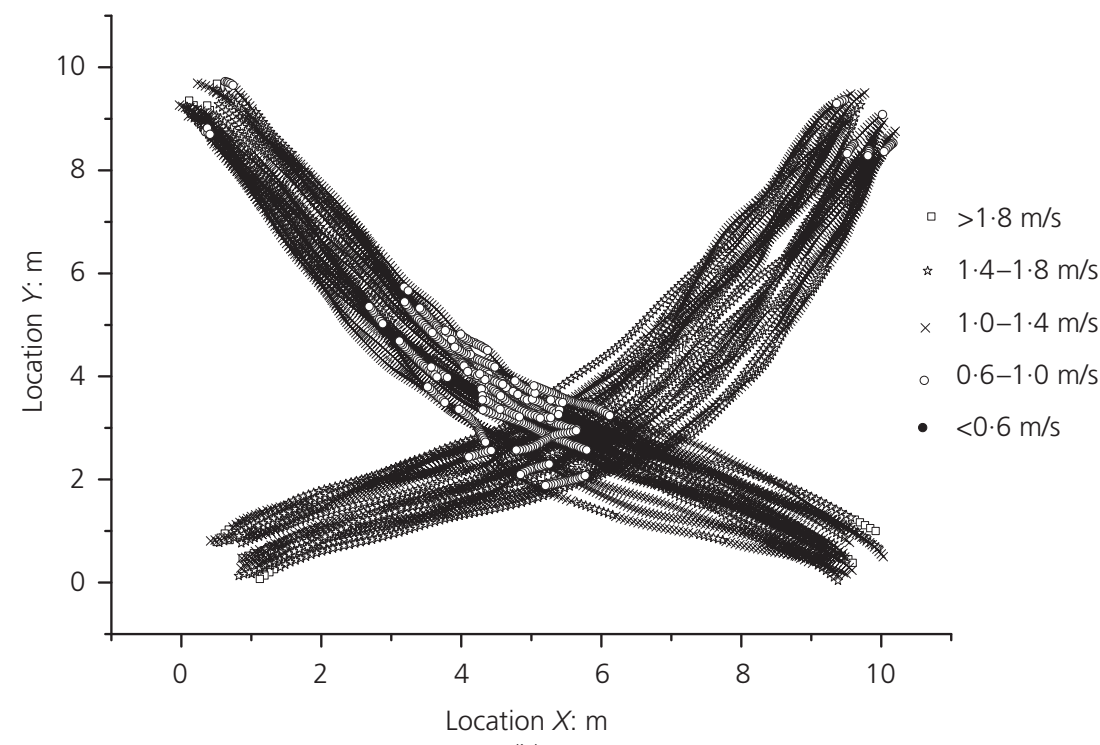

(b)

Figure 6. Comparison of pedestrian tracks with walking speed: (a) no guiding signage for two streams; (b) guiding signage added

roundabout. The entering and merging conflict of two streams is significant. By responding to the traffic signs, the pedestrians walk in the same direction. The bidirectional conflict on entry is eliminated and the merging conflict is alleviated. In summary, the benefit of adding auxiliary guiding signage has been observed through the experimental study.

\section{Case of three intersecting streams}

\subsection{Design of pedestrian experiment}

A three-stream intersection, which is a more complicated traffic phenomenon, is also observed at some subway stations. In this section, three additional experiments are described which studied the influence of a roundabout on such threestream intersections.

The experimental scenarios were established as in Figure 7. All the designs were the same as for the two-stream intersection flow, except that numbers of entrances and exits were changed to three. Three comparative experiments were designed as follows: the first one (scenario 5a) was a three-stream intersection with no roundabout; the second (scenario $5 \mathrm{~b}$ ) was a three-stream intersection with a roundabout; and the last one (scenario $5 \mathrm{c}$ ) included both a roundabout and traffic guiding signage. A pedestrian volume of $5000(\mathrm{p} / \mathrm{h}) / \mathrm{m}$ and roundabout radius of $1.0 \mathrm{~m}$ were employed in these experiments. 


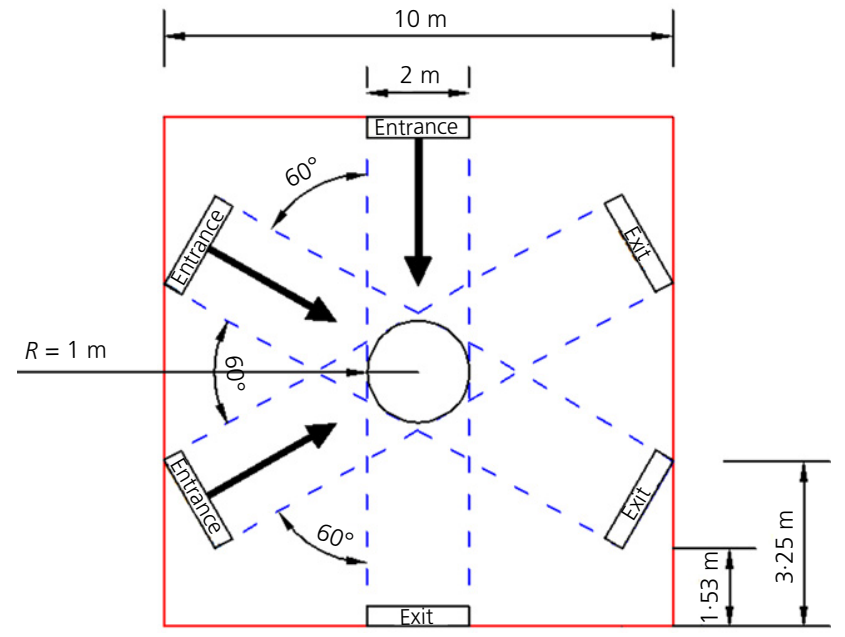

Figure 7. Experimental scenarios for three intersecting pedestrian traffic streams

\subsection{Data analysis}

The same set of performance indicators was used for the analysis. Speed, acceleration, walking distance and transit time for the three new scenarios are summarised in Table 6 . The walking distance for scenarios $5 \mathrm{~b}$ and $5 \mathrm{c}$ was increased $7 \cdot 54 \%$ and $10 \cdot 65 \%$, respectively, when compared with scenario $5 \mathrm{a}$. As regards transit time, there was no change between scenarios $5 \mathrm{a}$ and 5 b; a $9.09 \%$ decrease was seen for scenario $5 \mathrm{c}$. The mean speed for scenarios $5 \mathrm{~b}$ and $5 \mathrm{c}$ was significantly higher compared with that for scenario $5 \mathrm{a}$. The rates of increase were $22 \cdot 35 \%$ and $34 \cdot 12 \%$, respectively. In terms of the standard deviation of the speed, taking scenario $5 \mathrm{a}$ as the basis, decreases of $37 \cdot 74 \%$ and $49 \cdot 06 \%$ are seen for scenarios $5 \mathrm{~b}$ and $5 \mathrm{c}$, respectively. With respect to the standard deviation of acceleration, scenarios $5 \mathrm{~b}$ and $5 \mathrm{c}$ show decreases of $41.82 \%$ and $52.73 \%$, respectively, compared with scenario 5a. Overall, the walking efficiency is improved in scenarios $5 \mathrm{~b}$ and $5 \mathrm{c}$. Furthermore, scenario $5 \mathrm{c}$ shows higher efficiency than scenario $5 \mathrm{~b}$, which consolidates the finding from the previous section namely, that the signs have a positive impact in terms of improving pedestrian traffic flow. The three-stream scenarios were also compared with the two-stream scenarios. For all the performance indicators, the effect of the roundabout was more significant in the three-stream scenarios.
Figure 8 shows the pedestrian walking tracks for the three pedestrian streams scenarios. Conflict points (darkest areas) can be observed on all three scenarios. In terms of quantity of conflict points, it can be clearly seen that scenario 5a has the most and the scenario $5 \mathrm{c}$ has the least. When there is no roundabout (scenario 5a), three streams intersect near one point, which is near the centre of the experimental field. For scenario $5 \mathrm{~b}$, pedestrians walk around the roundabout and conflicts are separated into different locations. For scenario $5 \mathrm{c}$, where signs are posted, the only merging conflict is observed for the one-way counterclockwise flow. In this scenario, the conflict is minimised because the walking direction is the same.

In summary, the experimental results obtained from three pedestrian streams consolidate the findings from the experiments with two pedestrian streams. Adding a roundabout and guiding signs is useful to improve the traffic efficiency. This improvement is more significant for three pedestrian streams than for two pedestrian streams.

\section{Conclusions and recommendations}

In this study, the influence of installing a roundabout on the characteristics of pedestrian flow is investigated by conducting a series of experiments on pedestrians. It was found that installing a roundabout at a pedestrian intersection improves the traffic efficiency and reduces conflict for both two streams and three streams of pedestrian traffic flow. The size of the roundabout designed shows a positive relationship with the traffic efficiency in this study. In addition, the role of the roundabout is more important when the volume of pedestrians is large. Furthermore, setting guiding signs in front of the roundabout leads to the pedestrians walking in the same direction, which eliminates the initial bidirectional conflicts and reduces merging conflicts. Overall, it is recommended for subway management to install roundabouts at pedestrian intersections. However, it should be noted that this study has focused only on a balanced flow scenario. In other words, the volume of each stream of pedestrians in a multiple stream has been assumed as the same. To mimic the real-world situation fully, additional study using an unbalanced flow ratio is recommended. In addition, the characteristics of four intersecting pedestrian streams are significantly more complicated than for those in the current study, so it would be useful to analyse the effect of a roundabout in the case of four intersecting streams.

Table 6. Parameters analysis for different roundabout designs and traffic guiding signage

\begin{tabular}{|c|c|c|c|c|c|c|c|}
\hline \multirow[b]{2}{*}{ Scenario } & \multicolumn{2}{|c|}{ Facility setting } & \multirow[b]{2}{*}{$\begin{array}{l}\text { Transit } \\
\text { time: s }\end{array}$} & \multirow[b]{2}{*}{$\begin{array}{l}\text { Walking } \\
\text { distance: } \mathrm{m}\end{array}$} & \multirow[b]{2}{*}{$\begin{array}{c}\text { Mean speed: } \\
\mathrm{m} / \mathrm{s}\end{array}$} & \multirow[b]{2}{*}{$\begin{array}{c}\text { STD speed: } \\
\mathrm{m} / \mathrm{s}\end{array}$} & \multirow[b]{2}{*}{$\begin{array}{c}\text { STD acceleration: } \\
\mathrm{m} / \mathrm{s}^{2}\end{array}$} \\
\hline & $\begin{array}{l}\text { Radius of } \\
\text { roundabout: } m\end{array}$ & $\begin{array}{l}\text { Traffic guiding } \\
\text { signage }\end{array}$ & & & & & \\
\hline Scenario $5 a$ & None & None & 22 & $12 \cdot 20$ & 0.89 & 0.53 & $1 \cdot 10$ \\
\hline Scenario $5 b$ & 1 & None & 22 & $13 \cdot 12$ & 1.04 & 0.33 & 0.64 \\
\hline Scenario $5 c$ & 1 & Included & 20 & $13 \cdot 50$ & $1 \cdot 14$ & 0.27 & 0.52 \\
\hline
\end{tabular}


Transport

Volume 171 Issue TR1
Pedestrian roundabout improvement

strategy in subway stations

Sun, Hao, Gong, Qiu and Chen

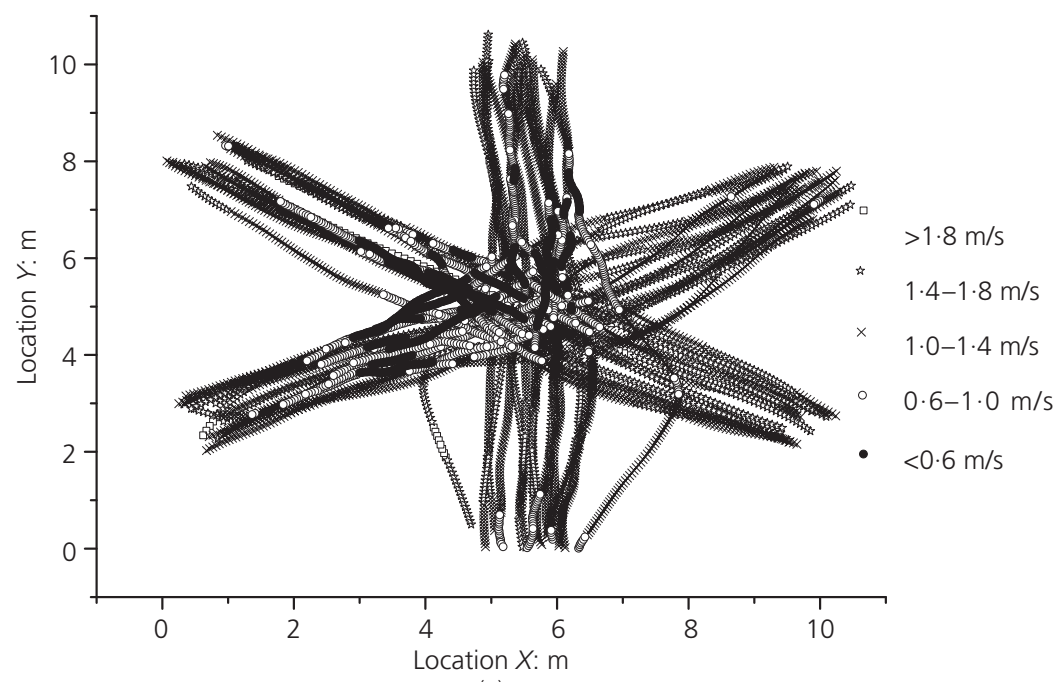

(a)

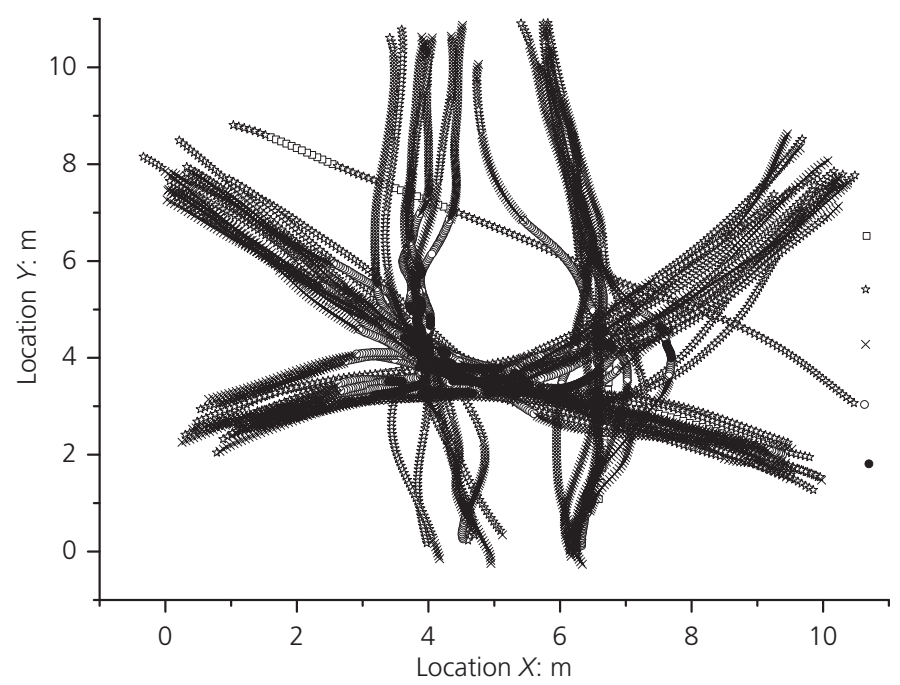

(b)

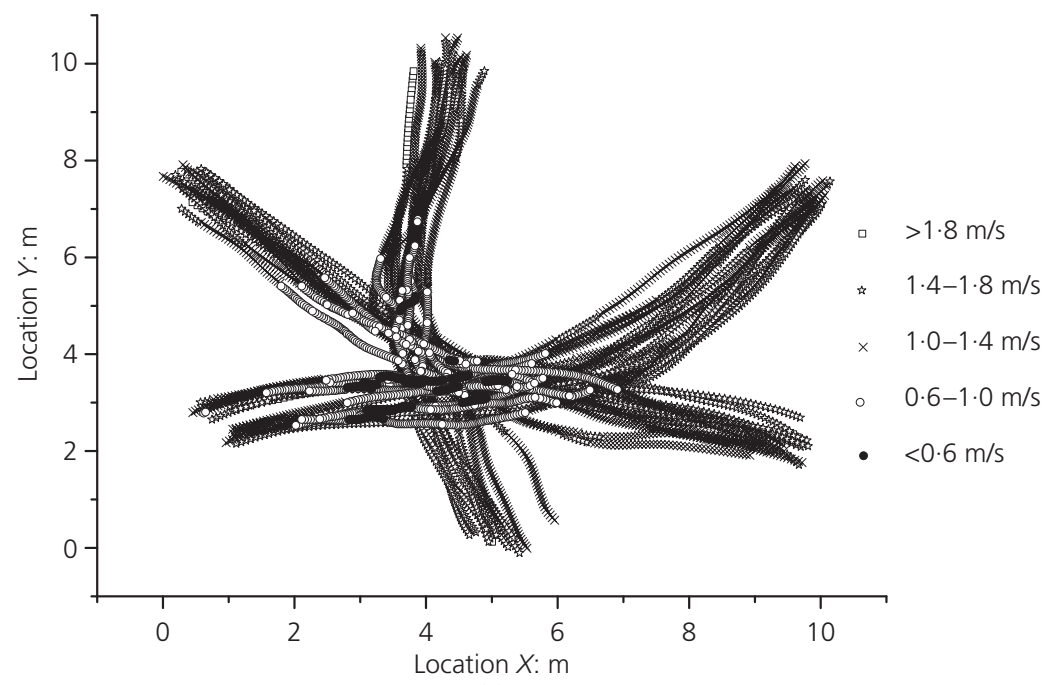

(c)

Figure 8. Pedestrian tracks with walking speed of: (a) scenario 5a; (b) scenario 5b; (c) scenario $5 c$ 


\section{Acknowledgements}

The authors would like to acknowledge the financial support for this study provided by the National Natural Science Foundation of China (no. 51308017), Beijing Nova Program (grant no. Z141106001814110), the Science and Technology Program of Beijing (grant no. D161100005616001) and the Funding Project for Academic Human Resources Development in Institutions of Higher Learning under the Jurisdiction of Beijing Municipality and Construction Technology Project of Ministry of Transport of the People's Republic of China (grant no. 2015318 J37 130).

\section{REFERENCES}

Ando K, Ota H and Oki T (1988) Forecasting the flow of people. Railway Research Review 45(8): 8-14.

Duparcmeur YL, Herrmann H and Troadec JP (1995) Spontaneous formation of vortex in a system of self motorised particles. Journal de Physique I 5(9): 1119-1128.

Dzubiella J and Löwen H (2002) Pattern formation in driven colloidal mixtures: tilted driving forces and re-entrant crystal freezing. Journal of Physics Condensed Matter 14(40): 9383-9395.

Guo RY, Wong SC, Huang HJ, Zhang P and Lam WHK (2010) A microscopic pedestrian-simulation model and its application to intersecting flows. Physica A Statistical Mechanics \& its Applications 389(3): 515-526.

Hediyeh H, Sayed T, Zaki MH and Ismail K (2012) Before and after analysis of pedestrian crossing speed behavior at scramble phase signalized intersections. Proceeding of the Transportation Research Board 91st Annual Meeting, The National Academies of Sciences, Engineering, and Medicine, Washington, DC, USA.

Helbing D (1996) Traffic modeling by means of physical concepts. In Traffic and Granular Flow (Wolf DE, Schreckenberg M and Bachem A (eds)). World Scientific, Singapore, pp. 87-104.

Helbing D (1997) Verkehrsdynamik. Springer, Berlin/Heidelberg, Germany (in German).

Helbing D (1998) A mathematical model for the behavior of individuals in a social field. Social Science Electronic Publishing 19(3): 189-219.

Helbing D and Molnar P (1997) Self-organization phenomena in pedestrian crowds. In Self-Organization of Complex Structures: From Individual to Collective Dynamics (Schweitzer F (ed.)). Gordon and Breach Science, Amsterdam, the Netherlands, pp. $560-577$.
Helbing D, Molnár P, Farkas IJ and Bolay K (2001) Self-organizing pedestrian movement. Environment \& Planning B Abstract 28(3): 361-383.

Helbing D, Buzna L, Johansson A and Werner T (2005) Self-organized pedestrian crowd dynamics: experiments, simulations, and design solutions. Transportation Science 39(1): 1-24.

Hoogendoorn S and Daamen W (2005) Self-organization in pedestrian flow. In Traffic \& Granular Flow '03 (Hoogendoorn SP, Luding S, Bovy PHL, Schreckenberg M and Wolf DE (eds)). Springer, Berlin/Heidelberg, Germany, pp. 373-382.

Hoogendoorn S and Piet HLB (2003) Simulation of pedestrian flows by optimal control and differential games. Optimal Control Applications \& Methods 24(3): 153-172.

Kittelson \& Associates, TRB (Transportation Research Board), US FTA (United States Federal Transit Administration), TCRP (Transit Cooperative Research Program) and TDC (Transit Development Corporation) (2003) Transit Capacity and Quality of Service Manual, 2nd edn. Transportation Research Board, Washington, DC, USA, Report 100

Mauro R (2010) Calculation of Roundabouts: Capacity, Waiting Phenomena and Reliability. Springer, Berlin/Heidelberg, Germany. Molnar P (1996) Modellierung und simulation der Dynamik von Fußgängerströmen. Shaker, Aachen, Germany (in German).

Sun L, Yang Z, Rong J et al. (2014) Study on the weaving behavior of high density bidirectional pedestrian flow. Mathematical Problems in Engineering 26(1): 1-9.

Tollazzi T, Tesoriere G, Guerrieri M et al. (2015a) Environmental, functional and economic criteria for comparing 'target roundabouts' with one- or two-level roundabout intersections. Transportation Research Part D, Transport \& Environment 34(1): 330-344.

Tollazzi T, Mauro R, Guerrieri M et al. (2015b) Comparative analysis of four new alternative types of roundabouts: "turbo," "flower," "target" and "four-flyover" roundabout. Periodica Polytechnica Civil Engineering 60(1): 51-56.

Usher JM and Strawderman L (2008) Emergent crowd behavior from the microsimulation of individual pedestrians. In Proceedings of the 2008 Industrial Engineering Research Conference (Fowler J and Mason S (eds)). Institute of Industrial Engineers, Norcross, GA, USA, pp. 673-678.

Wong SC, Asce M, Leung WL et al. (2010) Bidirectional pedestrian stream model with oblique intersecting angle. Journal of Transportation Engineering 136(3): 234-242.

Xie S, Wong SC, Lam WHK and Chen A (2013) Development of a bidirectional pedestrian stream model with an oblique intersecting angle. Journal of Transportation Engineering 139(7): 678-685.

\section{How can you contribute?}

To discuss this paper, please email up to 500 words to the editor at journals@ice.org.uk. Your contribution will be forwarded to the author(s) for a reply and, if considered appropriate by the editorial board, it will be published as discussion in a future issue of the journal.

Proceedings journals rely entirely on contributions from the civil engineering profession (and allied disciplines). Information about how to submit your paper online is available at www.icevirtuallibrary.com/page/authors, where you will also find detailed author guidelines. 